\title{
Multi Wavelength Low Level Lasers Transmeatal Irradiation (MWLLLTI) for Motion Sickness
}

\author{
Yuke Ma, Xiangang $\mathrm{Xu}^{*}$ \\ Institute of Crystal Materials, Shandong University, Jinan, China \\ Email: 201620262@mail.sdu.edu.cn, ^xxu@sdu.edu.cn
}

How to cite this paper: Ma, Y.K. and Xu, X.G. (2019) Multi Wavelength Low Level Lasers Transmeatal Irradiation (MWLLLTI) for Motion Sickness. Open Access Library Journal, 6: e5239.

https://doi.org/10.4236/oalib.1105239

Received: February 11, 2019

Accepted: February 22, 2019

Published: February 25, 2019

Copyright $\odot 2019$ by author(s) and Open Access Library Inc.

This work is licensed under the Creative Commons Attribution International License (CC BY 4.0).

http://creativecommons.org/licenses/by/4.0/

\section{(c) (i) Open Access}

\begin{abstract}
This was a case report which described the background, method, and test results of the effect of 3 wavelength lasers transmeatal irradiation treatment on motion sickness, using combined lasers with an output of $2.5 \mathrm{~mW} @ 532 \mathrm{~nm}$ +10.0 mW@808 nm + $2.0 \mathrm{~mW} @ 1064$ nm for transmeatal irradiation treatment. 10 patients aged $50 \pm 25$ were treated with MWLLLTI at a dose of 104 Joule in total for both ears per day for 15 consecutive days. It was found that MWLLLTI stimulation was effective in motion sickness treatment.
\end{abstract}

\section{Subject Area}

Otorhinolaryngology

\section{Keywords}

Low Level Laser Therapy, Multi Wavelength Low Level Laser Transmeatal Irradiation, Motion Sickness

\section{Introduction}

Motion sickness is a syndrome that happens when someone is subjected to certain kinds of motion [1]. Persons who response to a motion stimulus severely during travel may suffer from motion sickness. According to an investigation conducted via questionnaire survey about the motion sickness in China [2], motion sickness takes a high portion even in the young passenger populations; it is estimated about over $10 \%$. The syndrome symptoms range from uncomfortable feelings to completely incapacitating illness. Usually, these symptoms will occur within several minutes when experiencing motion and can sustain for several hours after their cessation. 
The pathogenesis of motion sickness is not clearly understood, but it is thought that the vestibular end-organs, the semicircular canals and otolith organs play an important role in causing motion sickness [3].

Over-the-counter medications such as cinnarizine/stugeron and prescription medications such as dramamine dimenhydrinate, scopolamine bonine/antivert and promethazine are readily available. Cinnarizine is not available in the United States, as it is not approved by the FDA [4]. All these medications often have side effects; however, over $75 \%$ patients do not know the toxic and side effects about the medicines they take.

Low level laser therapy (LLLT) is a laser source treatment that uses light of a monochromatic wavelength to promote tissue regeneration, to reduce inflammation, and to relieve pain. Unlike many other medical laser procedures, such as operative lasers, LLLT emits no heat, sound, or vibration. Instead of producing a thermal effect, it is thought that LLLT works by stimulating photochemical reactions in cells. Although the exact mechanism of biological action is unknown, several theories have been proposed and include increased mitochondrial ATP production, enhanced cellular proliferation, increased cellular oxygenation, increased serotonin and endorphin production, stimulation of angiogenesis, and suppression of inflammatory cytokines [5].

In recent years, the use of LLLT has extended beyond the realms of pain and wound healing, and recent research supports its potential benefits in retinal disease, stroke, neurodegeneration, neuromuscular disorders, and memory and mood disorders. LLLT can exert effective, reproducible, and meaningful changes in the normal and dysfunctional nervous tissue. This may highlight the value of LLLT as a novel and useful paradigm to treat visual, neurological, and psychological conditions, and support that neuronal energy metabolism could constitute a major target for neurotherapeutics of the eye and brain [6].

LLLT irradiation refers to the use of red-beam or near-infrared lasers with a wavelength of $600-1100 \mathrm{~nm}$ and an output power of $1-500 \mathrm{~mW}$ [7]. However, not only oxyhemoglobin and deoxyhemoglobin have a strong absorption in green region on the wavelength axis, but also cytochrome $\mathrm{C}$ oxidase has the same behavior [8]. From the first law of photochemistry, the Grotthuss-Draper law states that light must be absorbed by a compound in order for a photochemical reaction to take place. Thus, this case report proposed to use combined tri-lasers, especially $532 \mathrm{~nm}, 808 \mathrm{~nm}$ as well as $1064 \mathrm{~nm}$ lasers, to treat the motion sickness conditions. LLLT is safe and has been used to treat both tinnitus [9] and allergic rhinitis [10].

\section{Case Report}

For a rapid identification of effectiveness of LLLT for a motion sickness, an initial trial of a small sampling test was done. Although no ethics committee was organized, informed consent was obtained from each participant (volunteers) prior to the start of the treatment. The volunteers are listed in Table 1. 
Patients. 10 patients aged $50 \pm 25$ among them 2 males, and 8 females. Motion sickness past history is over 8 years at least. The oldest female patient aged 75 suffered the disease (bus sickness) over 60 years. All the patients are treated with MWLLLTI.

Laser irradiation. The transmeatal laser medical device (manufactured by YouRay Hestia Co. Ltd.) is employed to do the irradiation treatment. The wavelength of the tri-wavelength lasers are $532 \mathrm{~nm}, 808 \mathrm{~nm}$, and $1064 \mathrm{~nm}$ respectively. The correspondent average output power is $2.5 \mathrm{~mW} @ 532 \mathrm{~nm}, 10.0 \mathrm{~mW} @$ $808 \mathrm{~nm}$, and $2.0 \mathrm{~mW} @ 1064 \mathrm{~nm}$. The lasers are formed by using a semiconductor laser diode $808 \mathrm{~nm}$ to pump a laser crystal Nd:YVO4 to produce a $1064 \mathrm{~nm}$ laser output then a nonlinear crystal KTP is utilized to double the $1064 \mathrm{~nm}$ laser to have a second harmonic generation $532 \mathrm{~nm}$ laser output. All the lasers are packaged in earplug, and are combined into one light beam and then expanded to a circular spot with a diameter of $6 \mathrm{~mm}$ at a distance of $8 \mathrm{~mm}$ via an objective lens. In this way, it is very simple and convenient to do the MWLLLTI. Figure 1 shows the setup of MWLLLTI at $532 \mathrm{~nm}, 808 \mathrm{~nm}$, and $1064 \mathrm{~nm}$. All the lasers are working in pulsed mode at a frequency of $300 \mathrm{~Hz}$, with duration of $20 \%$.

Table 1. Volunteers list.

\begin{tabular}{cccc}
\hline Number & Age & Gender & Remark \\
\hline 1 & 75 & Female & YouRay Hestia Co. Ltd. Staffs Relative \\
2 & 72 & Female & YouRay Hestia Co. Ltd. Staffs Relative \\
3 & 73 & Male & YouRay Hestia Co. Ltd. Staff's Relative \\
5 & 41 & Female & YouRay Hestia Co. Ltd. Staff s Relative \\
6 & 25 & Female & YouRay Hestia Co. Ltd. Staff \\
7 & 69 & Male & YouRay Hestia Co. Ltd. Staff s friend \\
8 & 49 & Female & YouRay Hestia Co. Ltd. Staff s friend \\
9 & 40 & Female & YouRay Hestia Co. Ltd. Staff s friend \\
10 & 38 & Female & YouRay Hestia Co. Ltd. Staff s friend \\
\hline
\end{tabular}

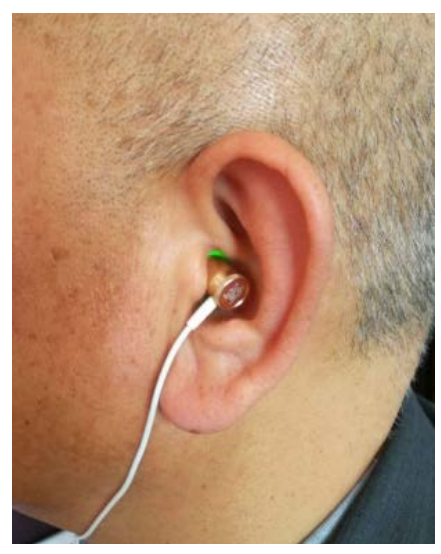

Figure 1. MWLLLTI treatment. 
When targeting the cochlea with laser light, it is essential to make sure that a sufficient amount of light reaches the target. Indeed, LLLT includes secondary effects through systemic mechanisms, but the energy at target is decisive for the biological effect [11]. A dose of 60 min (2 earplugs) per day gives energy of $18 \mathrm{~J}$ of $532 \mathrm{~nm}$ irradiation, $72 \mathrm{~J}$ of $808 \mathrm{~nm}$ irradiation, and $14 \mathrm{~J}$ of $1064 \mathrm{~nm}$ irradiation to the cochlea and the blood. A course of treatment takes 15 consecutive days and 60 min per day. Therapeutic evaluation protocols were set as immediately after the 15 days treatment, all the patients were tested by a bus ride.

It was found that MWLLLTI stimulation was safe and effective in motion sickness treatment. All of the volunteers were recovered. This effect remained for 2 weeks after the end of the treatment.

\section{Discussion}

According to our knowledge, this is the first report about using MWLLLTI to treat motion sickness successfully. The mechanism remains unclear. More samples may be needed to be treated to verify the further effectiveness. Multi wavelength low level lasers transmeatal irradiation is more beneficial as a new method for management of motion sickness.

\section{Conflicts of Interests}

No competing financial conflicts exist.

\section{References}

[1] Brainard, A. and Gresham, C. (2014) Prevention and Treatment of Motion Sickness. American Family Physician, 90, 41-46.

[2] http://www.sojump.com/report/5070950.aspx

[3] Shupak, A. and Gordon, C.R. (2006) Motion Sickness: Advances in Pathogenesis, Prediction, Prevention, and Treatment. Aviation, Space, and Environmental Medicine, 77, 1213-1223.

[4] https://en.wikipedia.org/wiki/Motion_sickness

[5] Huang, Y.-Y., Chen, A.C.-H., Carroll, J.D. and Hamblin, M.R. (2009) Biphasic Dose Response in Low Level Light Therapy. Dose-Response, Prepress.

https://doi.org/10.2203/dose-response.09-027.Hamblin

http://www.thorlaser.com/downloads/research/Biphasic-Dose-Response-in-Low-Le vel-Light-Therapy-Harvard.pdf

[6] Rojas, J.C. and Gonzalez-Lima, F. (2011) Low-Level Light Therapy of the Eye and Brain. Eye and Brain, 3, 49-67.

[7] Tiina, K. (1998) The Science of Low-Power Laser Therapy. Amsterdam, Gordan and Breach Science Publishers.

[8] http://www.ucl.ac.uk/medphys/research/borl/intro/spectra

[9] Demirkol, N., et al. (2017) Efficacy of Low-Level Laser Therapy in Subjective Tinnitus Patients with Temporomandibular Disorders. Photomedicine and Laser Surgery, 35, 1-5. https://doi.org/10.1089/pho.2016.4240

[10] Yaniv, E., Hadar, T., Shvero, J., Tamir, R. and Nageris, B. (2005) KTP/532 YAG 
Laser Treatment for Allergic Rhinitis. Journal of Allergy and Clinical Immunology, 115, 541-547.

[11] Tunér, J. and Hode, L. (2013) Letter to the Editor: Low-Level Laser on Hearing: Is There an Effect? ISRN Otolaryngology, 2013, Article ID: 839256. 